Research Article

\title{
Chaotic Vibration Prediction of a Free-Floating Flexible Redundant Space Manipulator
}

\author{
Congqing Wang and Linfeng $W u$ \\ College of Automation Engineering, Nanjing University of Aeronautics and Astronautics, Nanjing 210016, China \\ Correspondence should be addressed to Congqing Wang; cqwang@nuaa.edu.cn
}

Received 31 January 2016; Accepted 5 May 2016

Academic Editor: Mariano Artés

Copyright (c) 2016 C. Wang and L. Wu. This is an open access article distributed under the Creative Commons Attribution License, which permits unrestricted use, distribution, and reproduction in any medium, provided the original work is properly cited.

\begin{abstract}
The dynamic model of a planar free-floating flexible redundant space manipulator with three joints is derived by the assumed modes method, Lagrange principle, and momentum conservation. According to minimal joint torque's optimization (MJTO), the state equations of the dynamic model for the free-floating redundant space manipulator are described. The PD control using the tracking position error and velocity error in the manipulator is introduced. Then, the chaotic dynamic behavior of the manipulator is analyzed by chaotic numerical methods, in which time series, phase plane portrait, Poincaré map, and Lyapunov exponents are used to analyze the chaotic behavior of the manipulator. Under certain conditions for the joint torque optimization and initial values, chaotic vibration motion of the space manipulator can be observed. The chaotic time series prediction scheme for the space manipulator is presented based on the theory of phase space reconstruction under Takens' embedding theorem. The trajectories of phase space can be reconstructed in embedding space, which are equivalent to the original space manipulator in dynamics. The one-step prediction model for the chaotic time series and the chaotic vibration was established by using support vector regression (SVR) prediction model with RBF kernel function. It has been proved that the SVR prediction model has a good performance of prediction. The experimental results show the effectiveness of the presented method.
\end{abstract}

\section{Introduction}

Redundant space robots have been receiving an increasing attention in recent years because of their ability to perform high complexity tasks. Redundant robot's joint space dimension is greater than the task space dimension, which can overcome the shortcomings of low obstacle avoidance capacity and poor flexibility and dynamic performance. So it is of particular importance in the aerospace applications. However, the dynamic behavior of space robot systems is highly nonlinear, which in fact makes the design of controllers for such systems a challenge topic. A robust control strategy for free-floating space robots was presented in [1]. The strategy consists of a set of linear time invariant controllers coupled to the robotic manipulator through a set of high feedforward gains. The three-link space manipulator is modeled and simulated and the robustness of the controller has been demonstrated. An active damping control strategy could be used to reduce the structural vibrations of a space manipulator with flexible links during its on-orbit operations. An optimized adaptive vibration control via piezoelectric devices was proposed in [2]. To implement assembling, maintenance, repair, and contingency operations in space, the notion of free-flying space manipulator systems, in which robotic manipulators are mounted on a free-flying spacecraft, was discussed in [3]. The motion control of a free-floating space manipulator with parametric uncertainties and external disturbances was investigated and performed by adaptive nonlinear H1 controllers in [4]. In [5], the theoretical formulation and the experimental validation of two novel inverse kinematics solutions for redundant space manipulators aimed at locally minimizing the torque transferred to the spacecraft were presented. The first solution is based on a weighted Jacobian pseudoinverse and is derived by using Lagrange multipliers, whereas the second one is derived according to a constrained least squares approach by exploiting the 
linearity of the forward kinematics and the reaction torque dynamics equations. In [6], a novel composite sliding mode control was proposed to deal with the vibration suppression and trajectory tracking of a free-floating space rigid-flexible coupling manipulator with a rigid payload.

The flexible redundant space robot contains complex dynamic characteristics. The research has found that, based on the pseudoinverse control algorithm for the redundant robot, there are nonconservation and nonrepeatability in link movement. The structural flexibility inevitably causes the elastic deformation and vibration during the space manipulator's motion. However, the self-motion planning with redundancy to suppress vibration may result in chaotic vibration and joint angle drift phenomenon [7, 8].

Chaotic phenomena and chaotic behavior have been observed in numerous natural and model systems in physics. Chaotic dynamics appear frequently in nature and in nonlinear dynamical systems $[9,10]$. In [11], the nonlinear dynamic responses of the rotating continuous flexible shaft-rigid disk system with rub-impact between the disk and the stator were investigated. Time series, phase plane portrait, Poincaré map, bifurcation diagrams, and Lyapunov exponents were used to analyze the dynamic behavior of the system. In [12], an intelligent fuzzy chaotic structure controls the two-link robot arm by applying proper amplitude. This work presents the control and synchronization of chaos by designing the adaptive sliding mode controller [13]. In [14], based on MJTO, the state equations of chaos motion for the free-floating redundant space manipulator are established. The robust PD (proportional and derivative) compensatory control, the delayed feedback control, and the optimal control are designed in work space, joint space, and mode space respectively. The composite control can assure the trajectory tracking, chaotic suppression, and vibration control.

Although there exist some literatures on chaotic motions in Duffing's oscillator, inverted pendulum, and several other systems [15-17], there are very few works on the analysis of the nonlinear chaotic dynamic behavior of space robots. If chaos cannot be predicted and controlled, it might result in disaster and collapse a space robot system on many occasions. There is important significance to predict chaos motion for space robot security. Clearly, the ability to control chaos, that is, to convert the chaotic oscillation into the desired regular motion, would be beneficial.

Nonlinear time series prediction is increasingly used in many fields of engineering [18-20]. Recently, support vector machines (SVM) and support vector regression (SVR) based on statistical theory $[21,22]$ have become a key machine learning method and have been applied to deal with nonlinear time series prediction [23]. They have been proved to enhance the capability of prediction. For chaotic time series prediction, a novel direct prediction method based on echostate mechanisms and SVR was proposed in [24]. The method has been tested on both the benchmark problem (MackeyGlass time series) and real life time series (Wolf monthly sunspots number) and yearly runoff time series of the Yellow River.

In this paper, we propose an analytical approach to study the chaotic vibration in space manipulator. The prediction

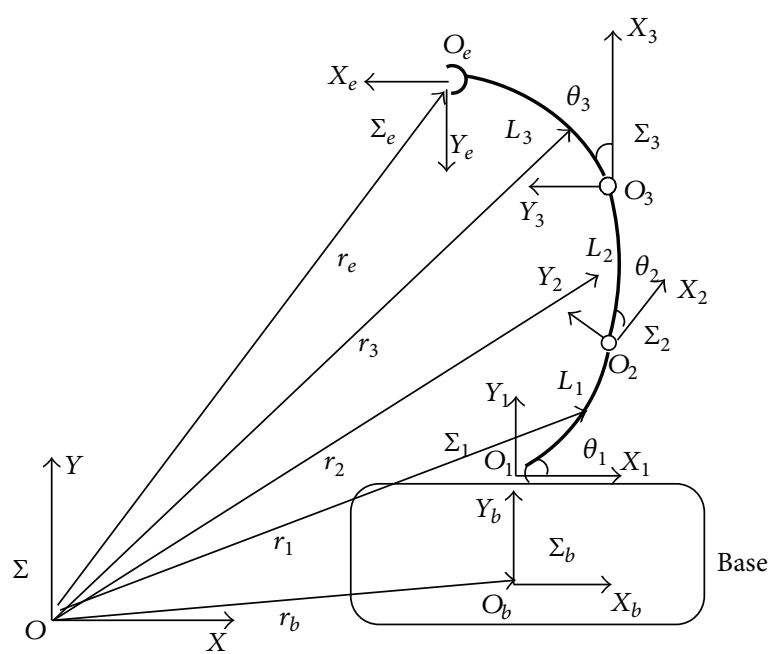

FIGURE 1: Free-floating space manipulator with three flexible links.

of chaotic motion is presented to improve the space robot performance and optimize the structure parameters on-orbit operations. The paper is organized as follows. In Section 2, the dynamic model for a planar redundant space manipulator with three flexible links is described. In Section 3, the phase space path graph changing over time and the time history of state variables are given in phase plane, and chaos phenomena can be determined by contrast, analysis, and synthesis. In Section 4 , the prediction of chaotic motion is performed by support vector regression based on the phase space reconstruction. In Section 5, the prediction results for the chaotic vibration motion of the system are shown and discussed. Finally, conclusions are given in Section 6.

\section{Dynamics Description of the Free-Floating Flexible Space Manipulator}

A planar structure diagram of the free-floating flexible space manipulator with the three flexible links is shown in Figure 1. The coordinate frames have been established for system modeling based on the Denavit-Hartenberg's tradition. Some parameters used in Figure 1 and in the rest of the paper are defined in Abbreviations.

The axial elongation of the links is ignored. Therefore, the links deflect only in a direction transverse to their length.

It is assumed that the longitudinal deformations of the links are negligibly small and Euler-Bernoulli beam theory is adequate to describe their flexible motions.

The following assumptions are made to derive a dynamical model of the space manipulator.

The system consists of a rigid body satellite and manipulator composed of three flexible links. External forces and torques are not applied to the system, and thus momentum conservation and equilibrium of forces strictly hold. The flexible motion of a link is described by a finite number of vibration modes which are constructed through component mode synthesis under the assumption of small defection. By 
the assumed mode technique and in the case of a pinnedpinned beam, the flexible link deflection can be expressed as

$$
\omega_{i}\left(x_{i}, t\right)=\sum_{j=1}^{n} \sin \left(\frac{j \pi x_{i}}{L_{i}}\right) Q_{i j}(t) \quad(i=1,2,3),
$$

where $n$ is the mode's order $(n=2)$ and $\sin \left(j \pi x_{i} / L_{i}\right)(i=$ $1,2,3)$ and $Q_{i j}(t)(i=1,2,3 ; j=1,2)$ are the mode shape functions and generalized modal-coordinates, respectively. $x_{i}$ is the coordinate along the longitudinal axis $X_{i}$ of the link $i$.

The position vectors of the arbitrary points of the links are, respectively, given by

$$
\begin{aligned}
& P_{1}(x, y)=\left(\begin{array}{c}
x_{1} C_{b 1}+\omega_{1} S_{b 1}+L_{b} C_{b}+x_{b} \\
x_{1} S_{b 1}-\omega_{1} C_{b 1}+L_{b} S_{b}+y_{b}
\end{array}\right), \\
& P_{2}(x, y)=\left(\begin{array}{c}
x_{2} C_{b 12}+\omega_{2} S_{b 12}+L_{1} C_{b 1}+L_{b} C_{b}+x_{b} \\
x_{2} S_{b 12}-\omega_{2} C_{b 12}+L_{1} S_{b 1}+L_{b} S_{b}+y_{b}
\end{array}\right), \\
& P_{3}(x, y) \\
& =\left(\begin{array}{c}
x_{3} C_{b 123}+\omega_{3} S_{b 123}+L_{2} C_{b 12}+L_{1} C_{b 1}+L_{b} C_{b}+x_{b} \\
x_{3} S_{b 123}-\omega_{3} C_{b 123}+L_{2} S_{b 12}+L_{1} S_{b 1}+L_{b} S_{b}+y_{b}
\end{array}\right) .
\end{aligned}
$$

The position vector of the end-effector is given by

$$
\begin{aligned}
P_{e} & (x, y) \\
& =\left(\begin{array}{c}
L_{3} C_{b 123}+L_{2} C_{b 12}+L_{1} C_{b 1}+L_{b} C_{b}+x_{b} \\
L_{3} S_{b 123}+L_{2} S_{b 12}+L_{1} S_{b 1}+L_{b} S_{b}+y_{b}
\end{array}\right),
\end{aligned}
$$

where $S_{b}=\sin \theta_{b}, S_{b 1}=\sin \left(\theta_{b}+\theta_{1}\right), S_{b 12}=\sin \left(\theta_{b}+\theta_{1}+\theta_{2}\right)$, $S_{b 123}=\sin \left(\theta_{b}+\theta_{1}+\theta_{2}+\theta_{3}\right), C_{b}=\cos \theta_{b}, C_{b 1}=\cos \left(\theta_{b}+\theta_{1}\right)$, $C_{b 12}=\cos \left(\theta_{b}+\theta_{1}+\theta_{2}\right)$, and $C_{b 123}=\cos \left(\theta_{b}+\theta_{1}+\theta_{2}+\theta_{3}\right)$.

The dynamic equations of the system are derived from the total kinetic energy and total potential energy of the system. The kinetic energy of the whole manipulator is related to the base and flexible links.

The kinetic energy of the entire system is given by

$$
\begin{aligned}
T & =T_{b}+\sum_{i=1}^{3} T_{i} \\
& =\frac{1}{2} I_{b} \dot{\theta}_{b}^{2}+\frac{1}{2} m_{b} \mathbf{V}_{b}^{2}+\frac{1}{2} \sum_{i=1}^{3}\left(I_{i} \dot{\theta}_{i}^{2}+\int_{0}^{L_{i}} \rho_{i} \mathbf{V}_{i}^{2} d x_{i}\right),
\end{aligned}
$$

where $T_{b}$ is the kinetic energy of the base, $T_{i}$ is the kinetic energy of the $i$ th link, $\rho_{i}$ is a line density of the $i$ th link, $\mathbf{V}_{b}, \mathbf{V}_{i}$ are linear velocities of the base and arbitrary point of the $i$ th link, respectively. $I_{b}, I_{i}$ are the inertia matrices of the base and the $i$ th link, respectively.

Potential energy of the elastic deformations of the links is expressed by

$$
U=\frac{1}{2} \sum_{i=1}^{3} \int_{0}^{L_{i}} \mathrm{EI}_{i}\left(\frac{\partial^{2} \omega_{i}\left(x_{i}, t\right)}{\partial x_{i}{ }^{2}}\right)^{2} d x_{i},
$$

where $\mathrm{EI}_{i}(i=1,2,3)$ is stiffness of flexible link.
According to D’Alembert-Lagrange, the dynamic equations of the manipulator are given by

$$
\begin{gathered}
\frac{d}{d t}\left[\frac{\partial L}{\partial \dot{\theta}_{i}}\right]-\frac{\partial L}{\partial \theta_{i}}=\tau_{i} \quad(i=1,2,3) \\
\frac{d}{d t}\left[\frac{\partial L}{\partial \dot{Q}_{i j}}\right]-\frac{\partial L}{\partial Q_{i j}}=0 \quad(i=1,2,3 ; j=1,2),
\end{gathered}
$$

where $L=T-\left(U_{1}+U_{2}+U_{3}\right)$ is a Lagrange function, $\theta_{i}(i=$ $1,2,3)$ is the joint angle, $\tau_{i}(i=1,2,3)$ is the joint torque, and $Q_{i j}(i=1,2,3 ; j=1,2)$ is the generalized coordinate of the flexible links.

The dynamic model is obtained by substituting the energy expressions (6) in Lagrange's equation. As a result, the dynamical equations can be written as

$$
\begin{gathered}
{\left[\begin{array}{lll}
M_{11} & M_{12} & M_{13} \\
M_{21} & M_{22} & M_{23}
\end{array}\right]\left[\begin{array}{c}
\ddot{\mathbf{X}}_{b} \\
\ddot{\boldsymbol{\theta}} \\
\ddot{\mathbf{Q}}
\end{array}\right]+\left[\begin{array}{lll}
D_{11} & D_{12} & D_{13} \\
D_{21} & D_{22} & 0_{6 \times 6}
\end{array}\right]\left[\begin{array}{c}
\dot{\mathbf{X}}_{b} \\
\dot{\boldsymbol{\theta}} \\
\dot{\mathbf{Q}}
\end{array}\right]} \\
+\left[\begin{array}{c}
0_{6 \times 6} \\
K
\end{array}\right] \mathbf{Q}=\left[\begin{array}{c}
\boldsymbol{\tau} \\
0_{6 \times 1}
\end{array}\right],
\end{gathered}
$$

where $M_{i j}(i=1,2, j=1,2,3)$ is the inertia matrix, $D_{i j}(i=$ $1,2, j=1,2,3)$ is the damping matrix, $K$ is a stiffness matrix, $\boldsymbol{\theta}=\left[\theta_{1}, \theta_{2}, \theta_{3}\right]^{T}, \mathbf{Q}=\left[Q_{11}, Q_{12}, Q_{21}, Q_{22}, Q_{31}, Q_{32}\right]^{T}$, and $\boldsymbol{\tau}=$ $\left[\tau_{1}, \tau_{2}, \tau_{3}\right]^{T}$ is the joint torque vector.

Since we assume the system is operated in free-floating mode without any external force and moment acting on the entire system, the linear momentum $\mathbf{P}$ and angular momentum $\mathbf{L}$ of the whole system are given by

$$
\begin{aligned}
& \mathbf{P}=m_{b} \mathbf{V}_{b}+\sum_{i=1}^{3} \int_{0}^{L_{i}} \rho_{i} \mathbf{V}_{i} d x_{i}, \\
& \mathbf{L}=I_{b} \dot{\boldsymbol{\theta}}_{b}+\mathbf{r}_{b} \times m_{b} \mathbf{V}_{b}+\sum_{i=1}^{3}\left(I_{i} \dot{\boldsymbol{\theta}}_{i}+\mathbf{r}_{i} \times m_{i} \mathbf{V}_{i}\right),
\end{aligned}
$$

where $m_{b}, I_{b}, m_{i}, I_{i}(i=1,2,3)$ are masses and inertia matrices of the base and the link, respectively, $\rho_{i}$ is a line density of the link $i(i=1,2,3), \mathbf{V}_{i}$ is the linear velocity of the arbitrary point of the $i$ th link, $I_{0}, I_{i}$ are the inertia matrices of the base and the $i$ th link, respectively, $\mathbf{V}_{b}$ and $\mathbf{V}_{i}$ are linear velocities of the base and the link, respectively, and $\mathbf{r}_{b}, \mathbf{r}_{c}$, and $\mathbf{r}_{i}$ are position vectors of the base, the end-effector, and the link, respectively.

At the beginning of this section we assumed that the space manipulator system is operated in free-floating mode so that total linear and angular momentum are conserved; we further assume that the total linear and angular momentum are zero. On the assumption that the total momentum is zero, the total momentum of the system can be written as

$$
A_{1} \dot{\mathbf{X}}_{b}+B_{1} \dot{\boldsymbol{\theta}}+D_{1} \mathbf{Q}+E_{1} \dot{\mathbf{Q}}=0
$$

where $A_{1}, B_{1}, D_{1}$, and $E_{1}$ are the coefficient matrices. 
We assumed that the planar space manipulator with an end-effector is mounted on a free-floating spacecraft. The end-effector velocity of the manipulator is expressed as

$$
\dot{\mathbf{X}}_{e}=J_{b} \dot{\mathbf{X}}_{b}+J_{q} \dot{\boldsymbol{\theta}}
$$

where $\dot{\mathbf{X}}_{e}=\left[\begin{array}{ll}\dot{x}_{e} & \dot{\mathrm{y}}_{e}\end{array}\right]^{T} \in R^{2 \times 1}$ is the velocity of the endeffector, $J_{b} \in R^{2 \times 3}$ is the Jacobian matrix for the end-effector relative to the base, and $J_{q} \in R^{2 \times 3}$ is the Jacobian matrix for the end-effector relative to the joint.

The dynamics and vibration equations of the space manipulator can be written as

$$
\begin{aligned}
& M_{11} \ddot{\mathbf{X}}_{b}+M_{12} \ddot{\boldsymbol{\theta}}+M_{13} \ddot{\mathbf{Q}}+D_{11} \dot{\mathbf{X}}_{b}+D_{12} \dot{\boldsymbol{\theta}}+D_{13} \dot{\mathbf{Q}} \\
& \quad=\boldsymbol{\tau}, \\
& M_{21} \ddot{\mathbf{X}}_{b}+M_{22} \ddot{\boldsymbol{\theta}}+M_{23} \ddot{\mathbf{Q}}+D_{21} \dot{\mathbf{X}}_{b}+D_{22} \dot{\boldsymbol{\theta}}+K \mathbf{Q}=0 .
\end{aligned}
$$

According to (9)-(11), the dynamic equation of the above space manipulator system is described as

$$
M \ddot{\boldsymbol{\theta}}+N=\boldsymbol{\tau},
$$

where $M$ is inertia matrix, $N=H_{1} \dot{\boldsymbol{\theta}}+H_{2} \mathbf{Q}+H_{3} \dot{\mathbf{Q}}$, and $H_{1}, H_{2}, H_{3}$ are coefficient matrices.

Utilizing (9) and (10), the relationship between joint velocities and end-effector velocities is

$$
\dot{\mathbf{X}}_{e}=J_{e} \dot{\boldsymbol{\theta}}+J_{r} \mathbf{Q}+J_{f} \dot{\mathbf{Q}}
$$

where $J_{e}, J_{r}, J_{f}$ are coefficient matrices.

\section{Dynamic Equations of the Space Manipulator Based on MJTO}

Joint torque optimization is essential to space manipulators, in which the electric power supply is limited. According to (13), the constrained equation for the manipulator can be expressed as

$$
\dot{J}_{e} \dot{\boldsymbol{\theta}}+J_{e} \ddot{\boldsymbol{\theta}}+\dot{J}_{r} \mathbf{Q}+J_{r} \dot{\mathbf{Q}}+\dot{J}_{f} \dot{\mathbf{Q}}+J_{f} \ddot{\mathbf{Q}}-\ddot{\mathbf{X}}_{e}=0 .
$$

Under certain conditions for the joint torque optimization and initial values, the above described system exhibits chaotic behavior. This section discusses the local optimization of the joint torque using classical Lagrange optimization technique.

The following local constrained optimization problem can be defined as

$$
\begin{array}{cl}
\min & E=\boldsymbol{\tau}^{T} \boldsymbol{\tau} \\
\text { subject to } & \dot{J}_{e} \dot{\boldsymbol{\theta}}+J_{e} \ddot{\boldsymbol{\theta}}+\dot{J}_{r} \mathbf{Q}+J_{r} \dot{\mathbf{Q}}+\dot{J}_{f} \dot{\mathbf{Q}}+J_{f} \ddot{\mathbf{Q}} \\
& -\ddot{\mathbf{X}}_{e}=0 .
\end{array}
$$

Using the Lagrange multiplier $\lambda \in R^{3 \times 1}$, the augmented cost function can be expressed as

$$
\begin{aligned}
\min \quad & \\
= & \boldsymbol{\tau}^{T} \boldsymbol{\tau} \\
& +\lambda^{T}\left(\dot{J}_{e} \dot{\boldsymbol{\theta}}+J_{e} \ddot{\boldsymbol{\theta}}+\dot{J}_{r} \mathbf{Q}+J_{r} \dot{\mathbf{Q}}+\dot{J}_{f} \dot{\mathbf{Q}}+J_{f} \ddot{\mathbf{Q}}-\ddot{\mathbf{X}}_{e}\right) .
\end{aligned}
$$

In order to determine the minimum solution to the unconstrained objective function, the derivatives of $E^{\prime}$ with respect to $\dot{\boldsymbol{\theta}}$ and $\boldsymbol{\lambda}$ must both vanish, so that we have

$$
\begin{aligned}
& \frac{\partial E^{\prime}}{\partial \ddot{\boldsymbol{\theta}}}=2 M^{T}(M \ddot{\boldsymbol{\theta}}+N)+J_{e}^{T} \boldsymbol{\lambda}=0, \\
& \frac{\partial E^{\prime}}{\partial \boldsymbol{\lambda}}=\dot{J}_{e} \dot{\boldsymbol{\theta}}+J_{e} \ddot{\boldsymbol{\theta}}+\dot{J}_{r} \mathbf{Q}+J_{r} \dot{\mathbf{Q}}+\dot{J}_{f} \dot{\mathbf{Q}}+J_{f} \ddot{\mathbf{Q}}-\ddot{\mathbf{X}}_{e}=0 .
\end{aligned}
$$

Then, we get

$$
\begin{aligned}
\ddot{\boldsymbol{\theta}}= & -0.5 B_{2}^{-1} A_{2}-0.5 B_{2}{ }^{-1} J_{e}^{T} \lambda \\
\boldsymbol{\lambda}= & \left(J_{e} B_{2}^{-1} J_{e}^{T}\right)^{-1}\left[-J_{e} B_{2}{ }^{-1} A_{2}\right. \\
& \left.+2\left(-\ddot{X}_{e}+\dot{J}_{e} \dot{\boldsymbol{\theta}}+\dot{J}_{r} Q+J_{r} \dot{Q}+\dot{J}_{f} \dot{Q}+J_{f} \ddot{Q}\right)\right],
\end{aligned}
$$

where $A_{2}=2 M N, B_{2}=M^{2}$, and $M=M^{T}$.

Substituting (19) into (18), we obtain

$$
\begin{aligned}
\ddot{\boldsymbol{\theta}}= & J_{B}^{+}\left[\ddot{\mathbf{X}}_{e}-\dot{J}_{r} \mathbf{Q}-J_{r} \dot{\mathbf{Q}}-\dot{J}_{f} \dot{\mathbf{Q}}-J_{f} \ddot{\mathbf{Q}}-\dot{J}_{e} \dot{\boldsymbol{\theta}}\right] \\
& -0.5\left[I-J_{B}^{+} J_{e}\right] B_{2}{ }^{-1} A_{2},
\end{aligned}
$$

where $J_{B}^{+}=B_{2}^{-1} J_{e}^{T}\left(J_{e} B_{2}^{-1} J_{e}^{T}\right)^{-1}$ is a weighted Moore-Penrose pseudoinverse and $I \in R^{3 \times 3}$ is an unit matrix. The second tern is possible to generate internal motions that reconfigure the manipulator structure without changing the end-effector position and orientation.

Equation (20) can be written as following the state equations:

$$
\begin{aligned}
& \dot{\mathbf{X}}=\mathbf{f}(\mathbf{X}, \mathbf{t})+\mathbf{B u}, \\
& \mathbf{Y}=\mathbf{C X},
\end{aligned}
$$

where $\mathbf{X}=\left[\mathbf{X}_{1}^{T}, \mathbf{X}_{2}^{T}\right]^{T}, \mathbf{X}_{1}=\boldsymbol{\theta}=\left[\theta_{1}, \theta_{2}, \theta_{3}\right]^{T}, \mathbf{X}_{2}=\dot{\boldsymbol{\theta}}=$ $\left[\dot{\theta}_{1}, \dot{\theta}_{2}, \dot{\theta}_{3}\right]^{T}, \mathbf{f}(\mathbf{X}, \mathbf{t})=\left[\mathbf{X}_{2}-\mathbf{J}_{B}^{+}\left[\dot{\mathbf{J}}_{r} \mathbf{Q}+\mathbf{J}_{r} \dot{\mathbf{Q}}+\dot{\mathbf{J}}_{f} \dot{\mathbf{Q}}+\mathbf{J}_{f} \ddot{\mathbf{Q}}+\dot{\mathbf{J}}_{e} \mathbf{X}_{2}\right]-\right.$ $\left.0.5\left[\mathbf{I}-\mathbf{J}_{B}^{+} J_{e}\right] \mathbf{B}_{2}^{-1} \mathbf{A}_{2}\right]^{T}, \mathbf{B}=\left[\begin{array}{ll}0_{3 \times 3} & J_{B}^{+}\end{array}\right]^{T}, \mathbf{C}=\left[\begin{array}{ll}I_{3 \times 3} & 0_{3 \times 3}\end{array}\right]^{T}$, and $\mathbf{u}=\ddot{\mathbf{X}}_{e}$.

The aim of the end-effector control law is to track the desired trajectory for the end-effector. $\mathbf{u}$ is the command acceleration vector. The proportional and derivative (PD) control can be introduced based on the state errors in the above system. The PD control law is chosen as follows:

$$
\ddot{\mathbf{X}}_{e}=\ddot{\mathbf{X}}_{\mathrm{ed}}+K_{v} \dot{\mathbf{e}}+K_{p} \mathbf{e}
$$

where $K_{p}, K_{v}$ are position and velocity gain matrices, respectively. $\mathbf{X}_{\mathrm{ed}}=\left[\begin{array}{ll}x_{\mathrm{ed}} & \mathrm{y}_{\mathrm{ed}}\end{array}\right]^{T}$ is the desired end-effector's trajectory. $\mathbf{X}_{e}=\left[\begin{array}{ll}x_{e} & \mathrm{y}_{e}\end{array}\right]^{T}$ is the actual end-effector's trajectory. $\mathbf{e}=$ $\mathbf{X}_{\mathrm{ed}}-\mathbf{X}_{e}$ and $\dot{\mathbf{e}}=\dot{\mathbf{X}}_{\mathrm{ed}}-\dot{\mathbf{X}}_{e}$ are defined as the tracking position error and velocity error respectively.

In this section, we investigate dynamical performance of the above system. The simulation results are obtained by using MATLAB. The step size is set to be 0.001 seconds. And simulation time is 50 seconds. The parameters of the planar 


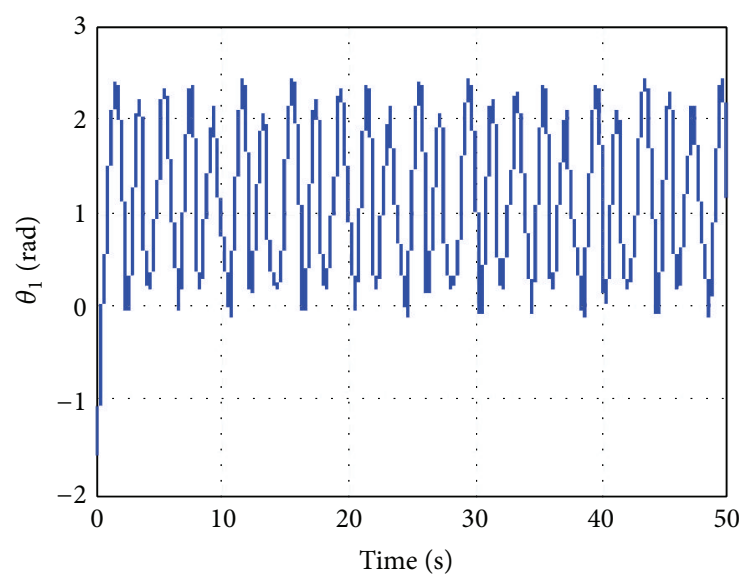

(a)

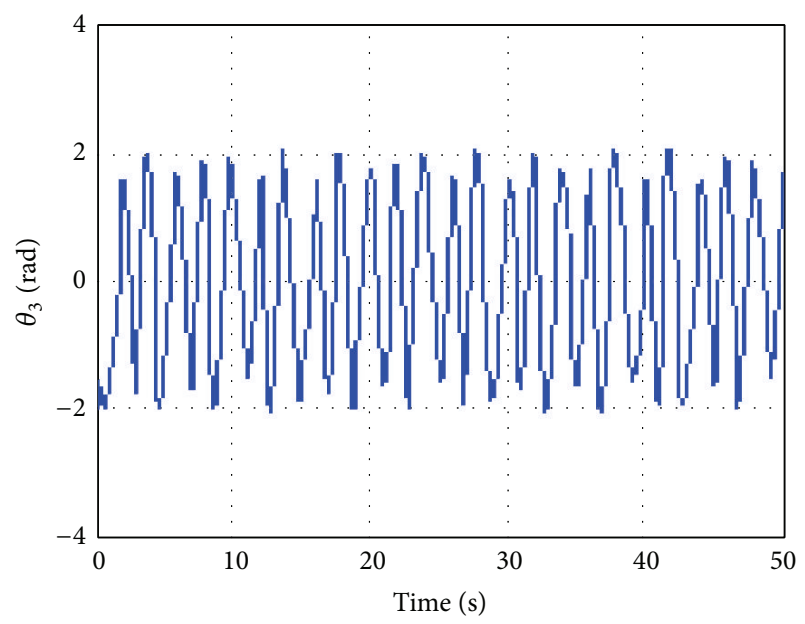

(c)

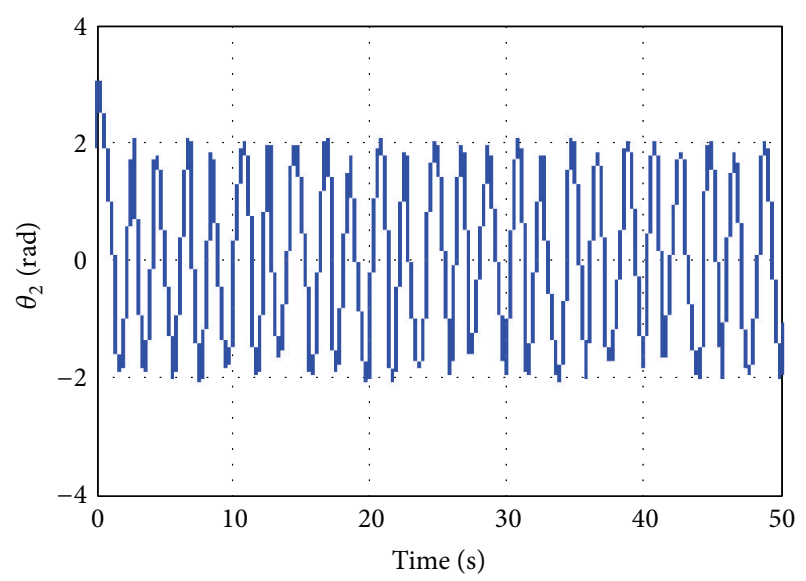

(b)

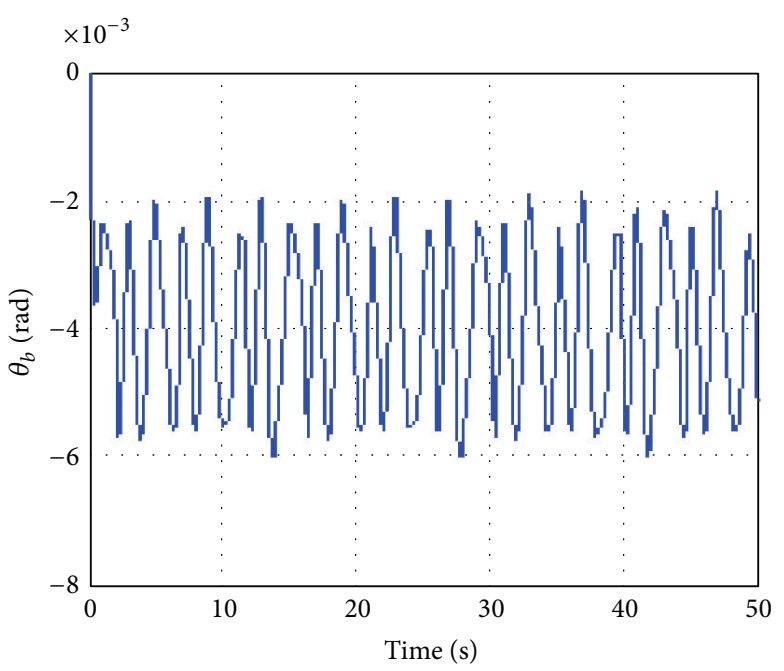

(d)

FIGURE 2: The time response of joints and base for the manipulator with PD control.

TABLE 1: Parameters of the space manipulator $(i=1,2,3)$.

\begin{tabular}{lcccc}
\hline Link $i$ & $m_{i}(\mathrm{~kg})$ & $L_{i}(\mathrm{~m})$ & $I_{i}\left(\mathrm{~kg} \cdot \mathrm{m}^{2}\right)$ & $\mathrm{EI}_{i}\left(\mathrm{~N} \cdot \mathrm{m}^{2}\right)$ \\
\hline Link 1 & 2 & 1 & 1.2 & 200 \\
Link 2 & 2 & 1 & 1.2 & 200 \\
Link 3 & 2 & 1 & 1.2 & 200 \\
The base & 2000 & 1.5 (radius) & 1000 & null \\
\hline
\end{tabular}

free-floating space redundant manipulator with 3 flexible links are given in Table 1.

In simulation, we can see that the free-floating redundant space manipulator in different initial configurations and $K_{v}$, $K_{p}$ which can guarantee the closed-loop stability may lead to chaos.

The original pose of the base is set to $X_{b}=$ $[0 m 0 m \text { orad }]^{T}$.

The desired trajectory for the end-effector is given by

$$
X_{\text {ed }}=[2+0.2 \cos (2 \pi t)(m) 2+0.2 \sin (2 \pi t)(m)]^{T} .
$$

According to (22), let $K_{p}=\left(\begin{array}{cc}40 & 0 \\ 0 & 40\end{array}\right)$ and $K_{v}=\left(\begin{array}{cc}12 & 0 \\ 0 & 12\end{array}\right)$.
In this paper we only give a set of simulation results. We consider $\boldsymbol{\theta}=[-\pi / 2,3 \pi / 5,-\pi / 2]^{T}, \dot{\boldsymbol{\theta}}=[0,0,0]^{T}$, as the initial conditions for the system.

According to the simulation results of nonlinear differential equations of the system, as shown in Figures 2, 3, 4, and 5 , the phase space path graph changing over time and the time history of state variables are given in phase plane. Chaos phenomena can be determined by contrast, analysis, and synthesis. In phase space, the periodic motion corresponds to the closed curves, and the chaotic motion corresponds to random distribution of a certain region of the path.

It can be seen from the phase diagrams that the trajectories in a certain random distribution neither overlap nor intersect but repeatedly fold. It can be seen that the joint's motion performs the chaotic attractor. It can also be seen that there is a certain coverage area (domain of attraction) in which the short-term predictable and long-term unpredictable chaotic motion exist. Preliminarily judging, there is chaotic motion in dynamics of the space robot system.

The above Poincaré sections have the infinite number of discrete points. By the nature of the Poincaré section, we 


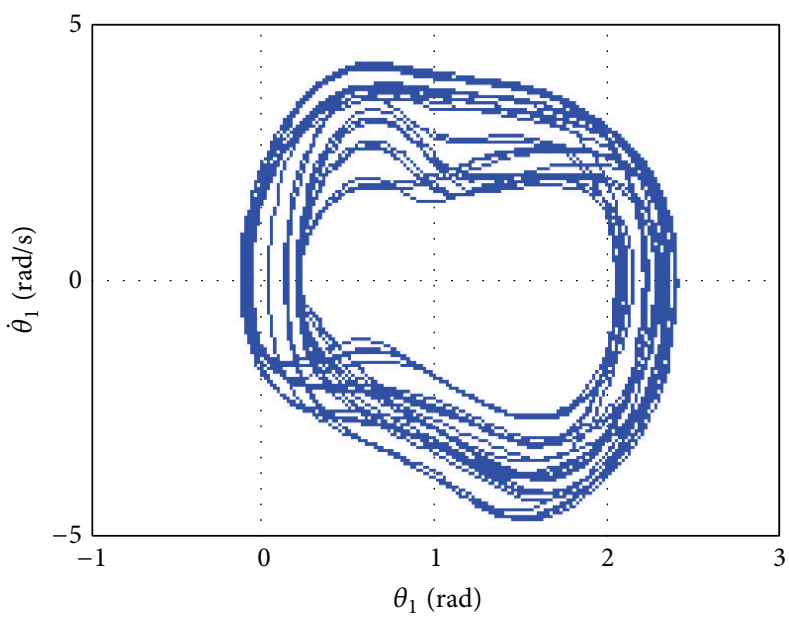

(a)

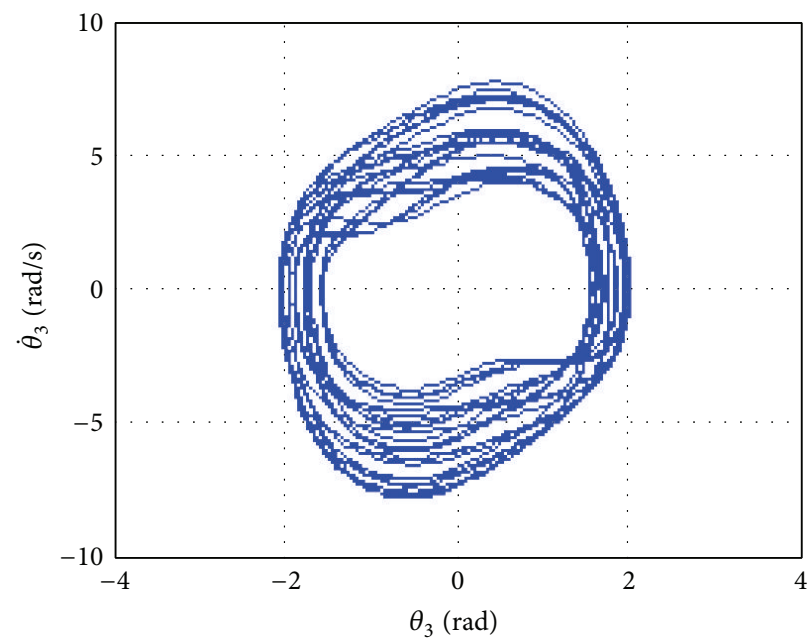

(c)

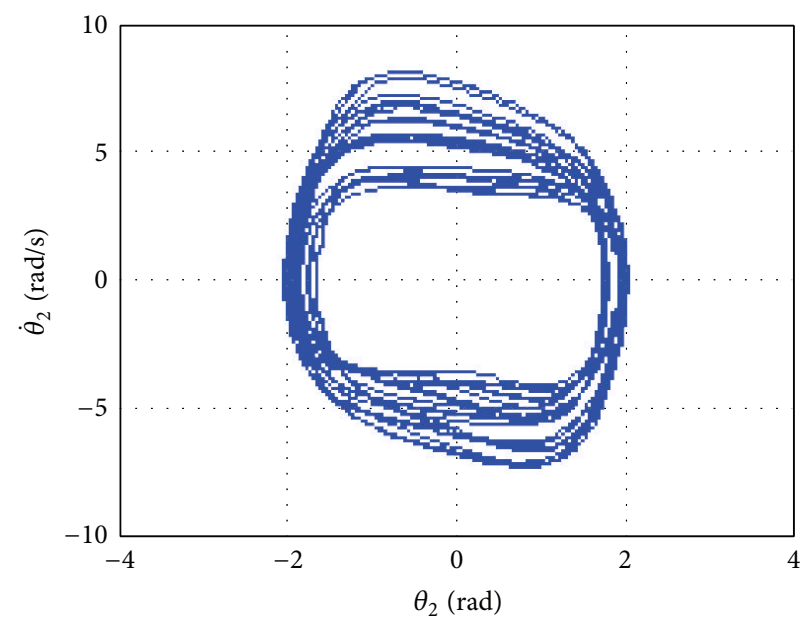

(b)

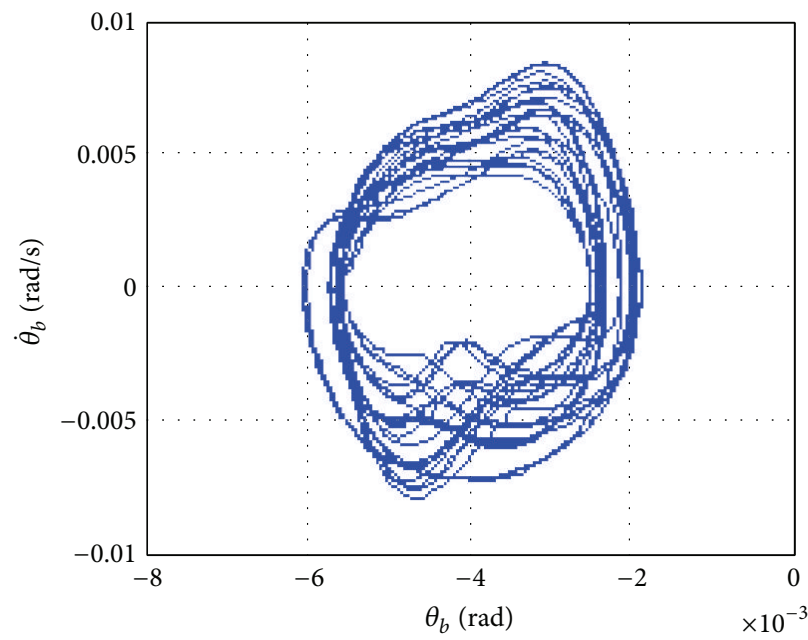

(d)

FIGURE 3: Phase plane trajectories of joints and base for the manipulator with PD control.

can see that the system has the chaotic characteristics. By means of small data sets arithmetic, the system's Lyapunov exponents for the time series are calculated as follows: $\lambda_{1}=$ $0.2183, \lambda_{2}=0.5412$, and $\lambda_{3}=0.0416$. The maximum Lyapunov exponent is positive, which demonstrates the chaotic motion in the space manipulator. Figure 5 shows the chaotic vibration curves of the generalized coordinate of the flexible links.

\section{Chaotic Prediction Based on SVR}

4.1. Phase Space Reconstruction. Chaotic time series prediction is based on the theory of phase space reconstruction under Takens' embedding theorem. A reconstructed phase space is $m$-dimensional metric space in which a time series is embedded. We can reconstruct a phase space trajectory from a single observation by a time delay. Given an observation chaotic time series $\{x(t)\}, t=1,2, \ldots, N$, selecting the embedding dimension $m$ and the delay time $\tau$, the phase space can be expressed as the following form:

$$
Y(t)=(x(t), x(t+\tau), \ldots, x(t+(m-1) \tau)),
$$

where $Y(t) \in R^{m}\left(t=1,2, \ldots, N_{\tau}\right)$ is a vector in the reconstruction phase space and $N_{\tau}=N-(m-1) \tau$ is the dimension of the vector in the reconstructed phase space. According to Takens' theorem, for proper embedding dimension $m$ and time delay $\tau$, the trajectory of phase space can be reconstructed in embedding space, which is equivalent to original system in dynamics. Therefore, $x(t+T)=f(Y(t))$ $(t=1,2, \ldots, N)$, where $T>0$ is the forward prediction step and $f(\cdot)$ is the reconstructed prediction model.

4.2. SVR Predicting Model for Chaotic Time Series. In this section, we briefly review the support vector regression (SVR) theory $[21,22]$. Given a training sample set $\left\{x_{k}, y_{k}, k=\right.$ $1,2, \ldots, N\}$, where $x_{k} \in R^{n}$ is the input vector and $y_{k} \in R$ is the desired output, the above training set can be mapped into 


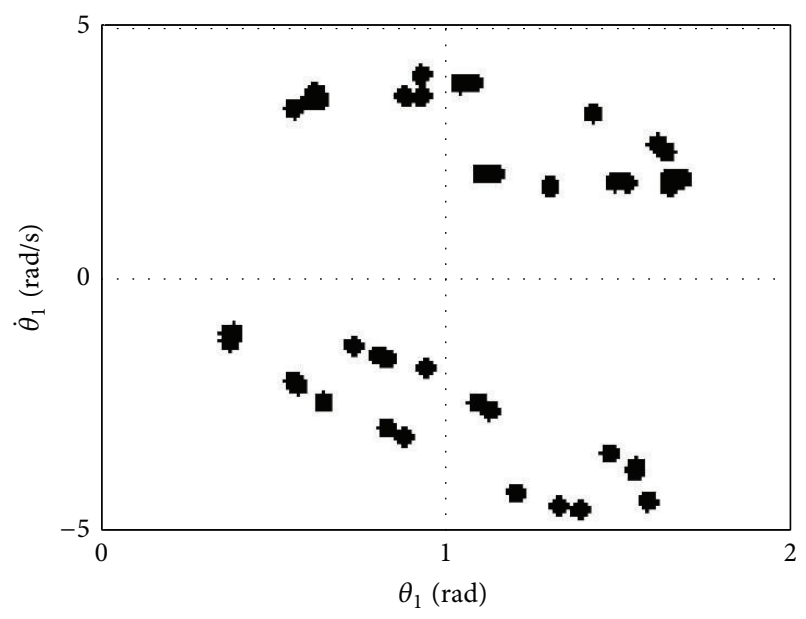

(a)

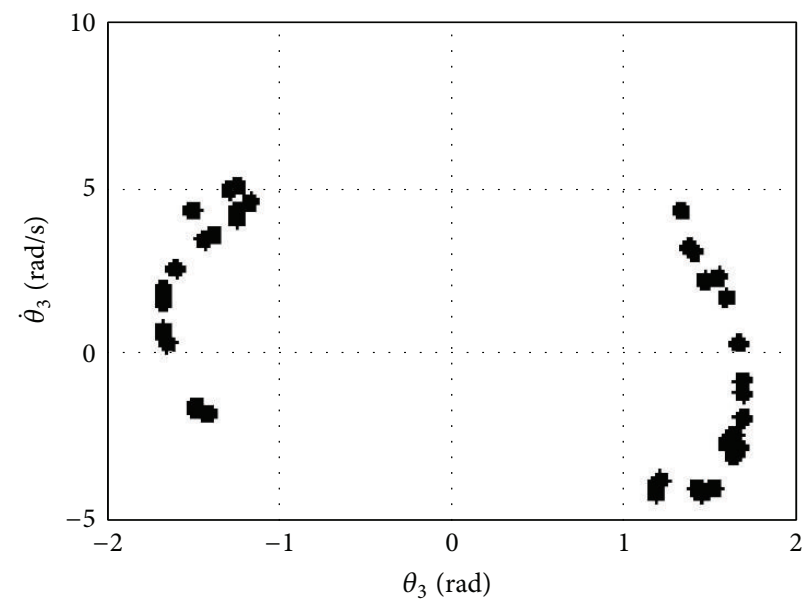

(c)

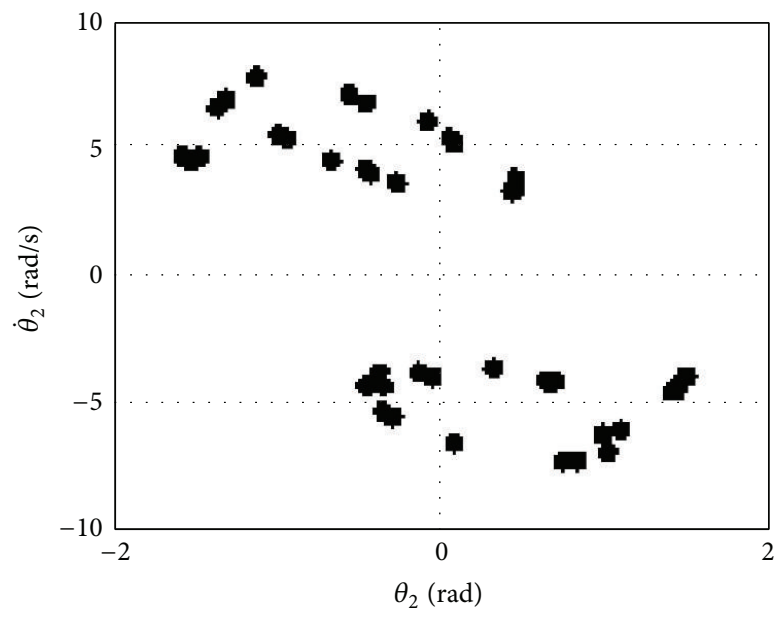

(b)

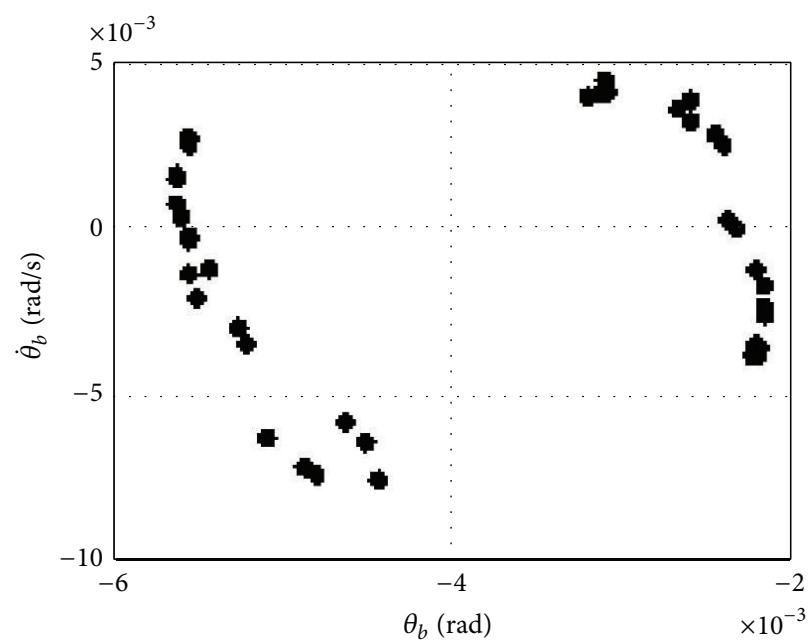

(d)

FIgURE 4: Poincaré sections of joints and base for the manipulator with PD control.

high dimension featured space, in which optimized linear regression function can be described as follows:

$$
f(x)=\omega^{T} \phi(x)+b,
$$

where $\omega \in R^{n}$ is a high-dimensional weight vector and the bias $b \in R$ is a scalar and $\phi(\cdot)$ is a nonlinear mapping from the input space to a feature space. For a nonlinear regression problem, $\varepsilon$-SVR model is based on Vapnik's $\varepsilon$-insensitive loss function for function approximation [21]. Given the definition of Vapnik's $\varepsilon$-insensitive loss function, we have

$$
\left|y_{k}-f(x)\right|_{\varepsilon}= \begin{cases}0, & \left|y_{k}-f(x)\right|_{\varepsilon} \leq \varepsilon \\ \left|y_{k}-f(x)\right|-\varepsilon, & \text { else }\end{cases}
$$

where $\varepsilon$ is the maximum value of tolerable error.
Therefore, by means of introduction of relaxation factors $\xi_{k}, \xi_{k}{ }^{*}$, the optimization problem can be formulated in the primal space as the following:

$$
\begin{gathered}
\min _{\omega, b, \xi, \xi^{*}} J\left(\omega, \xi, \xi^{*}\right)=\frac{1}{2}(\omega \cdot \omega)+C \sum_{k=1}^{N}\left(\xi_{k}+\xi_{k}^{*}\right) \\
\text { s.t } \quad y_{k}-\omega \cdot \varphi\left(x_{k}\right)-b \leq \varepsilon+\xi_{k}^{*} \\
-y_{k}+\omega \cdot \varphi\left(x_{k}\right)+b \leq \varepsilon+\xi_{k} \\
\xi_{k}, \xi_{k}^{*} \geq 0, k=1,2, \ldots, N,
\end{gathered}
$$

where $C$ is a punishment parameter.

The above optimization problem can be transformed into the following Lagrange dual form:

$$
\begin{array}{rl}
\min _{\alpha, \alpha^{*}} & L\left(\alpha, \alpha_{i}^{*}\right) \\
& =-\frac{1}{2} \sum_{k=1}^{N} \sum_{l=1}^{N}\left(\alpha_{k}-\alpha_{k}^{*}\right)\left(\alpha_{l}-\alpha_{l}^{*}\right) K\left(x_{k}, x_{l}\right)
\end{array}
$$



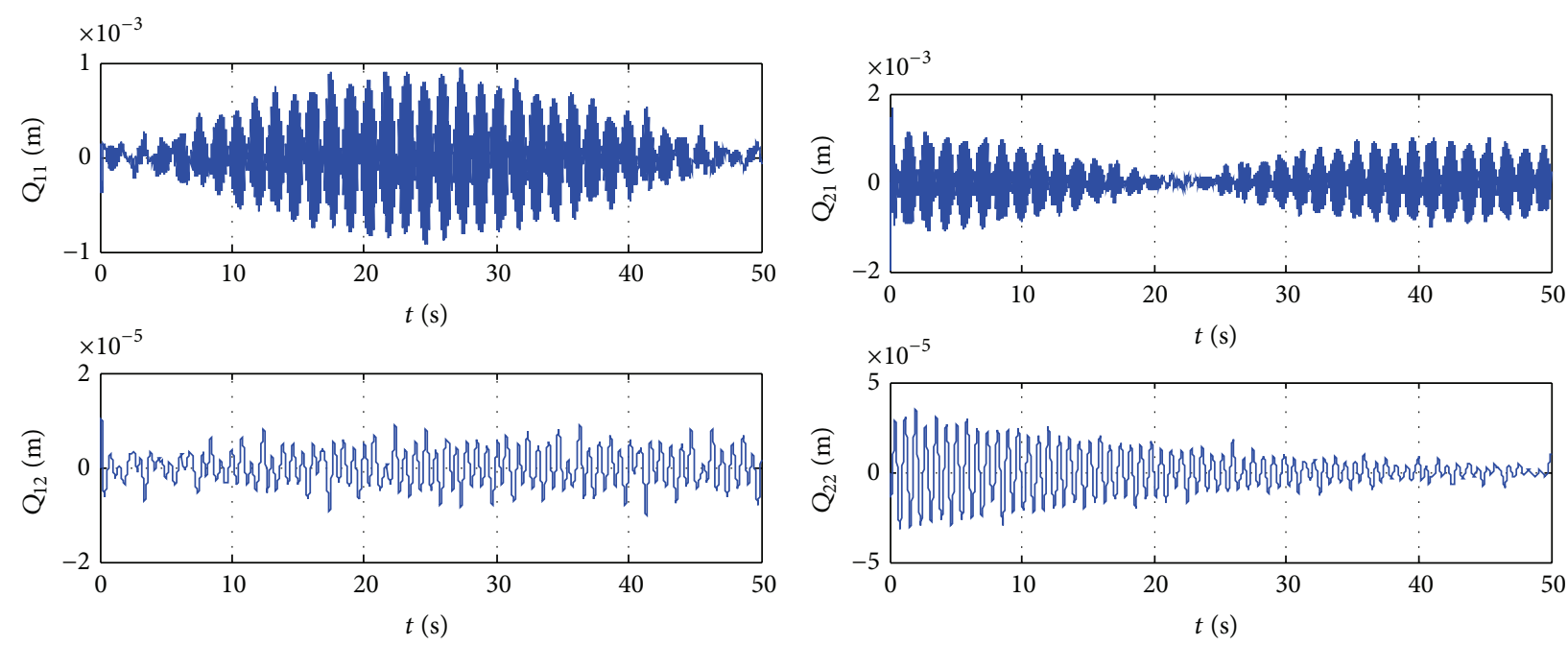

(a)

(b)
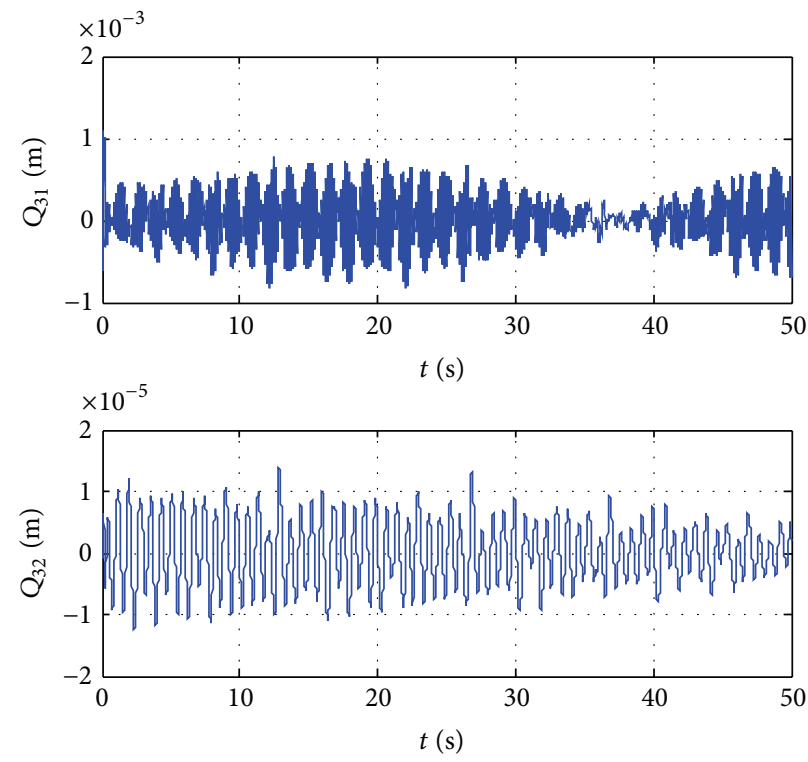

(c)

FIGURE 5: Chaotic vibration curves of the flexible links ((a) $Q_{11}, Q_{12}$, (b) $Q_{21}, Q_{22}$, and (c) $Q_{31}, Q_{32}$ ).

$$
\begin{array}{r}
-\varepsilon \sum_{k=1}^{N}\left(\alpha_{k}+\alpha_{k}^{*}\right)+\sum_{k=1}^{N} y_{k}\left(\alpha_{k}-\alpha_{k}^{*}\right) \\
\text { s.t } \quad \sum_{k=1}^{N}\left(\alpha_{k}-\alpha_{k}^{*}\right)=0, \quad \alpha_{k}, \alpha_{k}^{*} \in[0, C],
\end{array}
$$

where $\alpha_{k}$ and $\alpha_{k}^{*}$ are Lagrange multipliers and $K\left(x_{k}, x_{l}\right)=$ $\phi^{T}\left(x_{k}\right) \cdot \phi\left(x_{l}\right)$ is called the kernel function. The kernel function is defined as the following radial basis function:

$$
K\left(x_{k}, x_{l}\right)=\exp \left(-\frac{\left(x_{k}-x_{l}\right)^{T}\left(x_{k}-x_{l}\right)}{2 \sigma^{2}}\right),
$$

where $\sigma$ is the width of the function.
After the quadratic optimization problem with inequality constraints is solved, the parameters $\omega$ and $b$ in (28) are obtained:

$$
\begin{aligned}
& \omega=\sum_{k=1}^{N}\left(\alpha_{k}-\alpha_{k}^{*}\right) \phi\left(x_{k}\right), \\
& b=y_{k}-\sum_{l}^{N}\left(\alpha_{l}-\alpha_{l}^{*}\right) K\left(x_{k}, x_{l}\right)-\varepsilon, \quad \alpha_{k} \in(0, C) .
\end{aligned}
$$

Finally, the SVR function is obtained by (25) in the dual space:

$$
f(x)=\sum_{k=1}^{N}\left(\alpha_{k}-\alpha_{k}^{*}\right) K\left(x, x_{k}\right)+b .
$$

Setting $x(t+T)=f(Y(t))$ is the support vector regression corresponding to (32) and $T=1$, the one-step prediction 
TABLE 2: The prediction values of RMSRE for the joint angles of the link and attitude angle of the base.

\begin{tabular}{lcccc}
\hline & $\theta_{1}$ & $\theta_{2}$ & $\theta_{3}$ & $\theta_{b}$ \\
\hline Training data & $1.27 \times 10^{-7}$ & $1.18 \times 10^{-7}$ & $1.26 \times 10^{-7}$ & $1.22 \times 10^{-7}$ \\
Test data & $1.70 \times 10^{-7}$ & $1.27 \times 10^{-7}$ & $1.33 \times 10^{-7}$ & $1.36 \times 10^{-7}$ \\
\hline
\end{tabular}

TABLE 3: The prediction values of RMSRE for the chaotic vibration.

\begin{tabular}{lcccccc}
\hline & $Q_{11}$ & $Q_{12}$ & $Q_{21}$ & $Q_{22}$ & $Q_{31}$ & $Q_{32}$ \\
\hline Training data & $5.42 \times 10^{-5}$ & $1.33 \times 10^{-6}$ & $8.61 \times 10^{-5}$ & $9.80 \times 10^{-7}$ & $1.74 \times 10^{-4}$ & $1.73 \times 10^{-6}$ \\
Test data & $9.28 \times 10^{-5}$ & $2.82 \times 10^{-5}$ & $2.70 \times 10^{-4}$ & $1.24 \times 10^{-6}$ & $1.82 \times 10^{-4}$ & $1.99 \times 10^{-6}$ \\
\hline
\end{tabular}

model for chaotic time series based on SVM has the following form:

$$
\begin{aligned}
\widehat{x}(t+1) & =f(Y(t))=f(x(t), \ldots, x(t+(m-1) \tau)) \\
& =\sum_{k=1}^{N_{\tau}}\left(\alpha_{k}-\alpha_{k}^{*}\right) \phi(Y(t)) \cdot \phi\left(x_{k}\right)+b
\end{aligned}
$$

where $\widehat{x}(t+1)$ is the value of one-step forecasting; then the next point in phase space can be expressed as

$$
\begin{aligned}
& Y(t+1)=(\hat{x}(t+1), x(t+\tau), x(t+2 \tau), \ldots, \\
& \quad x(t+(m-2) \tau)) .
\end{aligned}
$$

\section{Simulation Results and Discussions}

The time series responses of the system states for the manipulator using PD control are shown in Figure 2. The 5000 data points as training and test samples from the raw data of chaotic motion in the space manipulator are collected and normalized to $[-0.50 .5]$. In order to eliminate the influence of the initial value, the initial 2000 points were discarded, the phase space reconstruction of the chaotic time series was done by the embedding dimension $m=3$, and the delay time $\tau=1$. Then 1500 points were taken as training data; 1500 points were taken as test data. The root mean square error (RMSE) was used as integral performance index to evaluate time series prediction errors between the predicted signal and its reference signal with the SVR model obtained. The RMSE is defined as

$$
\mathrm{RMSE}=\sqrt{\frac{1}{n} \sum_{i=1}^{n}\left(y_{i}-\hat{y}_{i}\right)^{2}}
$$

where $y_{i}$ is a real value, $\widehat{y}_{i}$ is a predicted value, and $n$ is the number of test points. The width ranges of Gaussian kernel $\sigma$ and parameters $C, \varepsilon$ are set to $2.8,2.2$, and 0.001 , respectively. The prediction experiment was done with the SVR model obtained. The prediction and absolute error curves for the chaotic time series of the manipulator are shown in Figures 6 and 7, respectively. It can be seen from Figure 6 that the actual time series responses of the links have perfect approximation to the predicted responses. Also, it can be seen from Figure 7 that the time series responses have drastic vibration for whole motion period and prediction errors show the undesirable peaks in some sample points.
The prediction values of RMSE for the chaotic time series as shown in Tables 2 and 3 are used for evaluating the performance of chaotic vibration prediction. The smaller the value of RMSE is, the closer the predicated values are to the real values.

The results of the prediction experiment show that the proposed prediction method has good prediction accuracy and successful evaluating performance in capturing and modeling the nonlinear chaotic dynamical behavior of this space manipulator.

\section{Conclusions}

In this paper, chaotic behavior of a planar space flexible manipulator was investigated. Time series, phase plane portrait, Poincaré map, and Lyapunov exponents were used to analyze the chaotic dynamic behavior of the manipulator. According to the largest Lyapunov exponent, phase plane portrait, and Poincaré map figures, the simulation results reveal the existence of chaotic motion in the space manipulator with three flexible links under the condition of MJTO. Based on complex dynamics inherent characteristic of chaotic and phase space reconstruction theory for the planar free-floating flexible redundant space manipulator, the training data of the time series can be obtained. And then a model based on least squares SVR (support vector regression) is proposed, which combines features of chaotic time series' development and change, and it obtains the training samples through phase space reconstruction of the time series by considering all the information from relative parameters' chaotic time series. Prediction experiments show that this method has good prediction accuracy and is an effective prediction method. How to control this chaos in the space manipulator will be our future research work.
Abbreviations
$\Sigma-O X Y$ :
$\Sigma_{e}-O_{e} X_{e} Y_{e}: \quad$ The frame of the end-effector
$\Sigma_{i}-O_{i} X_{i} Y_{i}: \quad$ The frame of the link $i(i=1,2,3)$
$\mathbf{X}_{b}=\left[x_{b}, y_{b}, \theta_{b}\right]^{T}$ : The pose of the base
$\mathbf{X}_{i}=\left[x_{i}, y_{i}, \theta_{i}\right]^{T}: \quad$ The pose of the link $L_{i}(i=1,2,3)$
$\boldsymbol{\theta}=\left[\theta_{1}, \theta_{2}, \theta_{3}\right]^{T}: \quad$ The vector of the joint angles
$\mathbf{r}_{b}$ : with reference to the inertia frame 

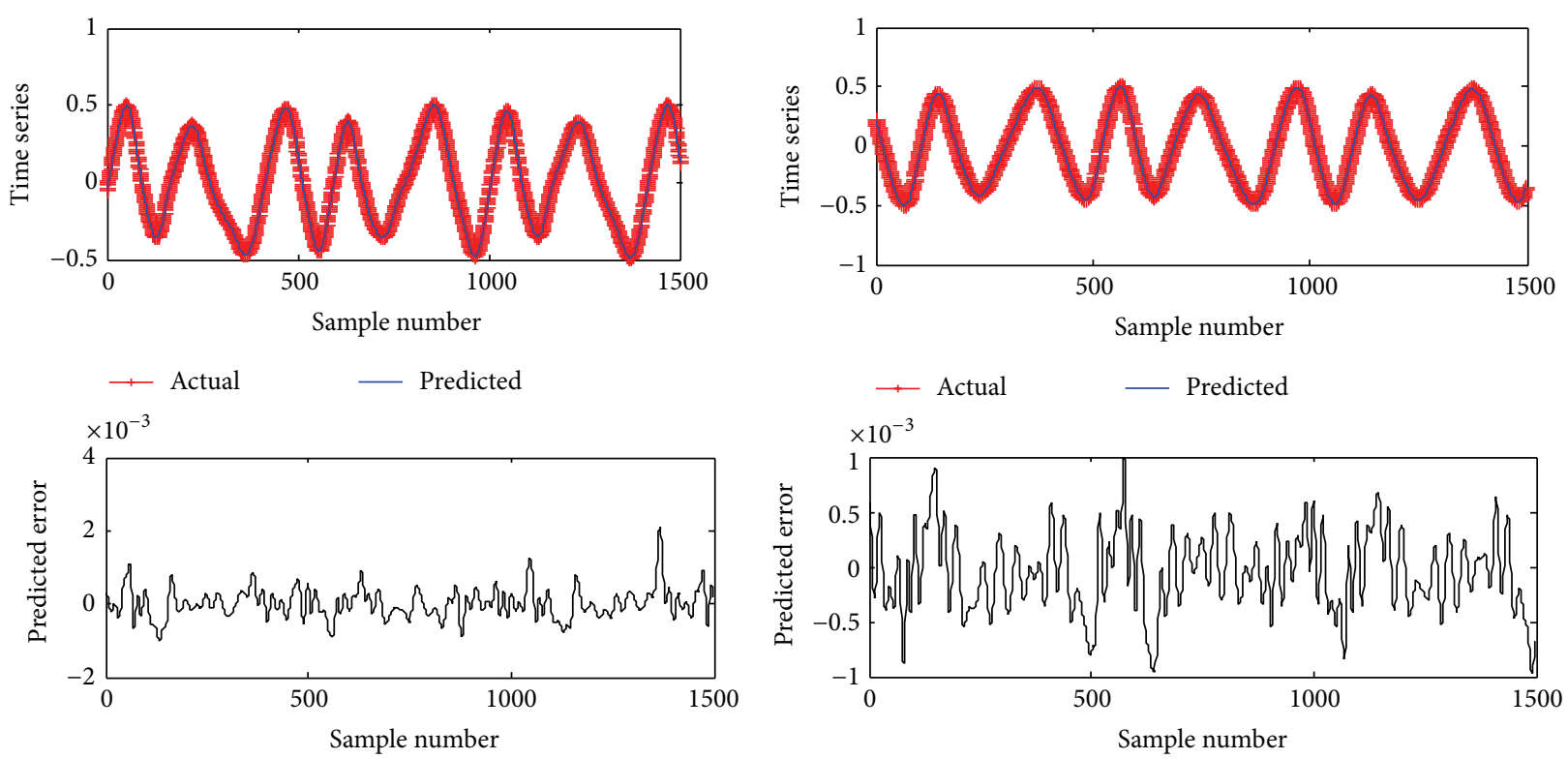

(a)

(b)
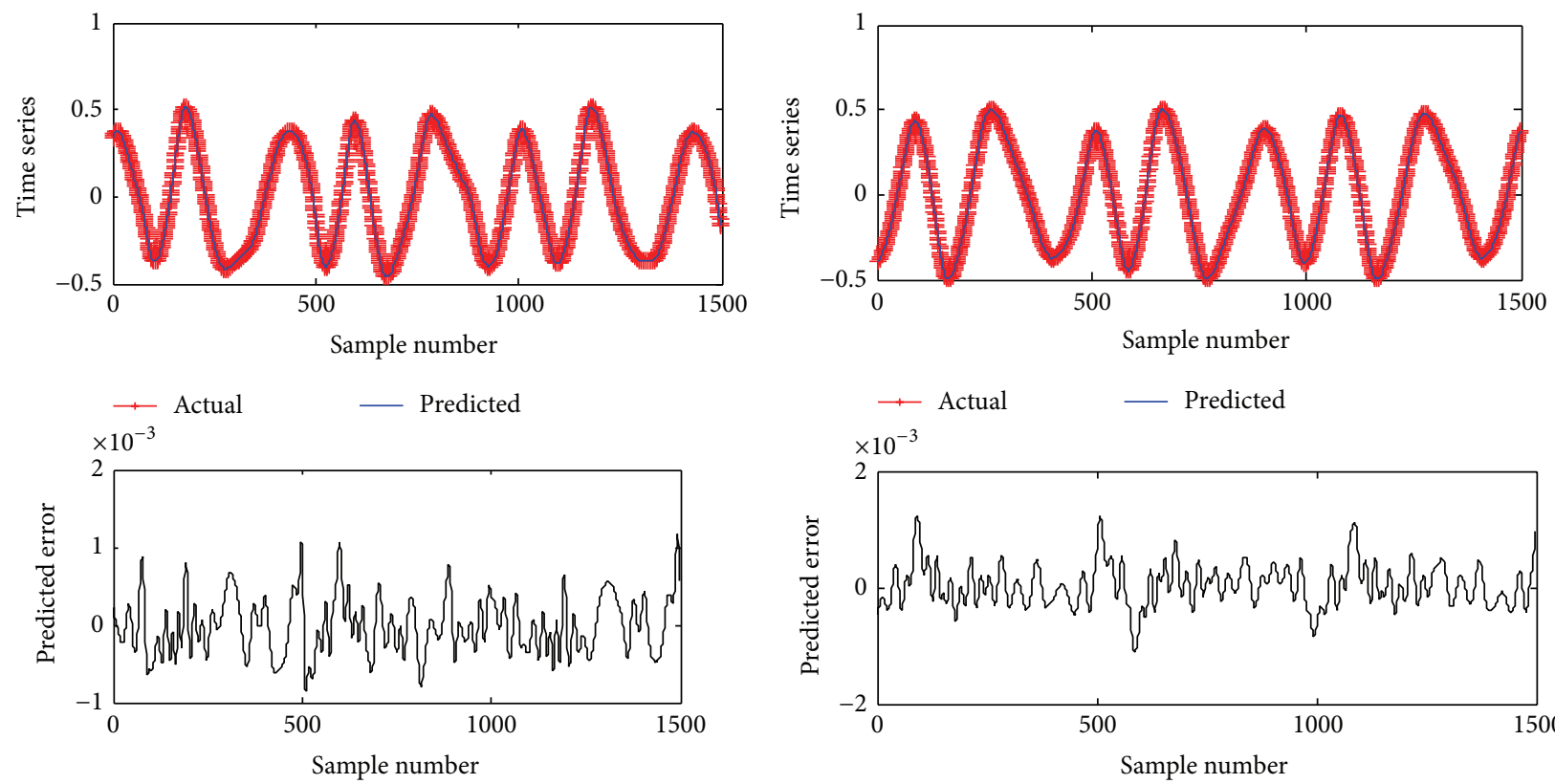

(c)

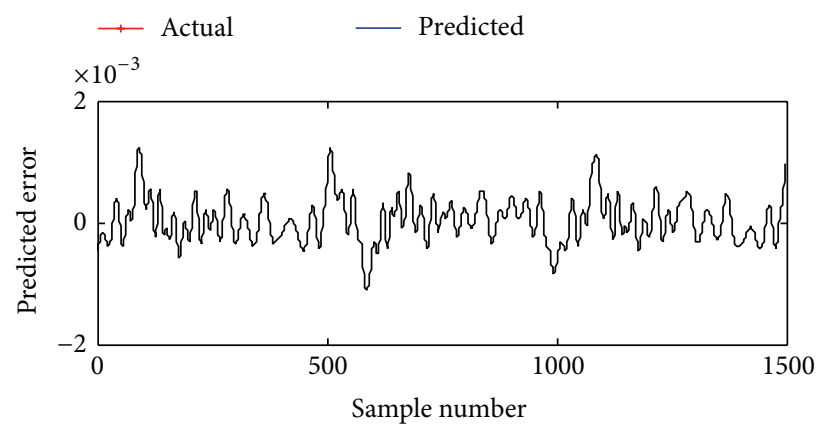

(d)

FIGURE 6: The prediction results of the chaotic time series ((a)-(c) $\theta_{1}, \theta_{2}, \theta_{3} ;$ (d) $\theta_{b}$ ).

$\mathbf{r}_{i}$ : The position vector of the $i$ th link with reference to the inertia frame

$\mathbf{r}_{c}$ : The position vector of the payload center with reference to the inertia frame

$\theta_{i}$ : Joint angle of the link $i(i=1,2,3)$

$\theta_{b}$ : Attitude angle of the base

$L_{i}$ : Length of the flexible link $i(i=1,2,3)$

$L_{b}$ : Radius of the base

$m_{i}$ : Mass of the link $i(i=1,2,3)$

$m_{b}$ : Mass of the base.

\section{Competing Interests}

The authors declare that they have no competing interests.

\section{Acknowledgments}

This work was supported by the Foundation of Manufacturing Systems Engineering State Key Laboratory, Xi'an Jiaotong University of China (no. 201002), and the Jiangsu Province Science and Technology Support Plan (no. BE2014712). 

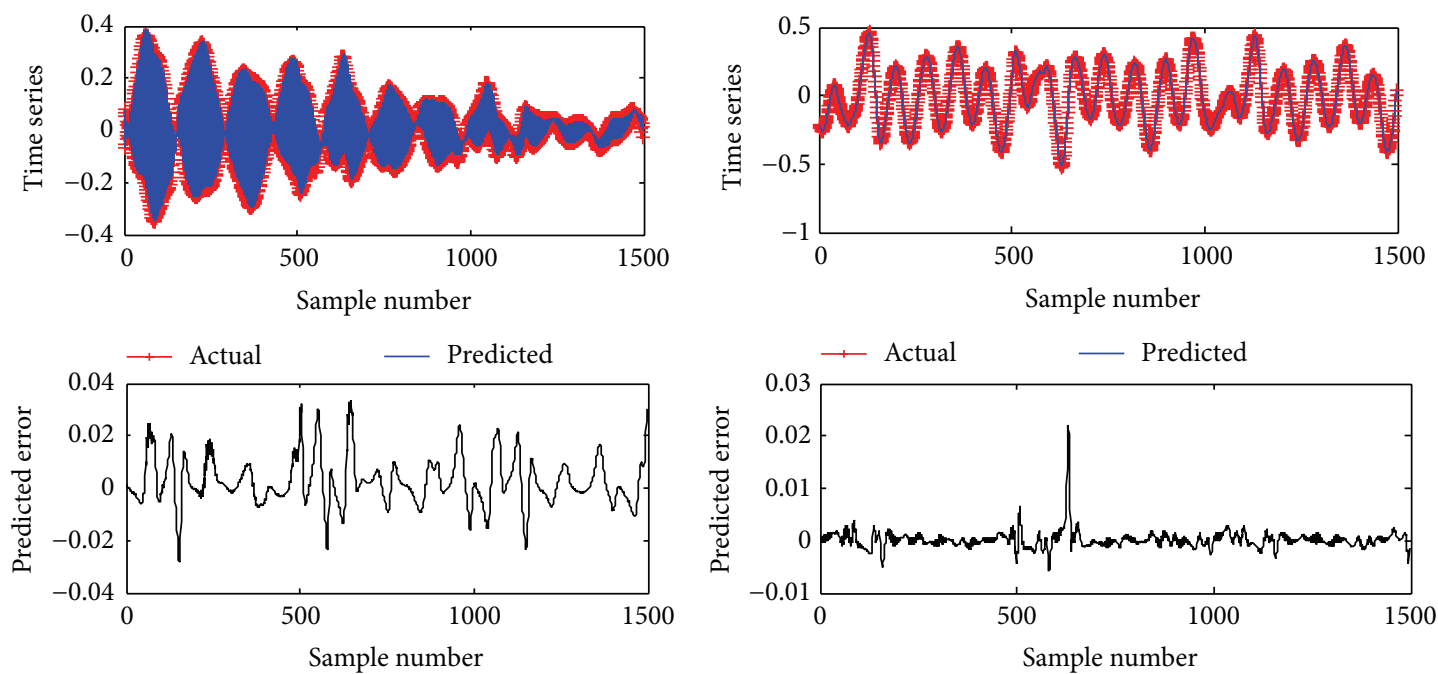

(a)

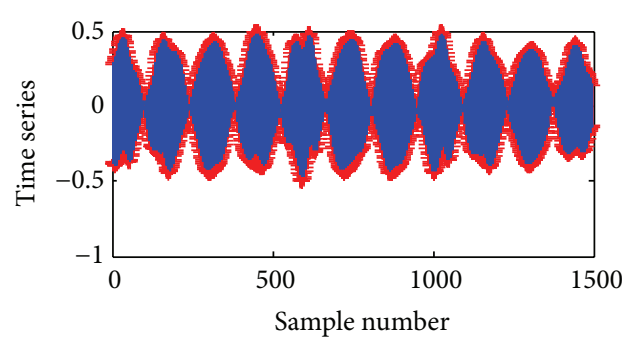

(b)

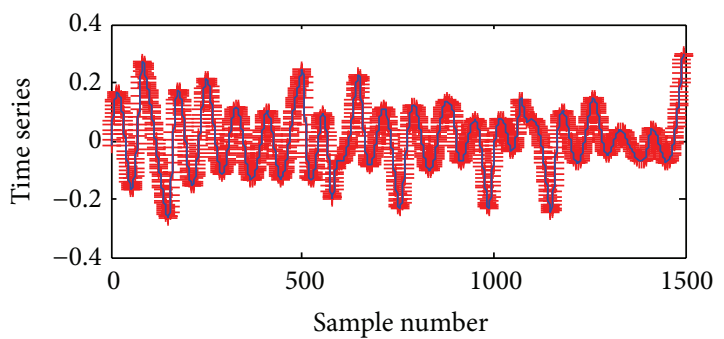

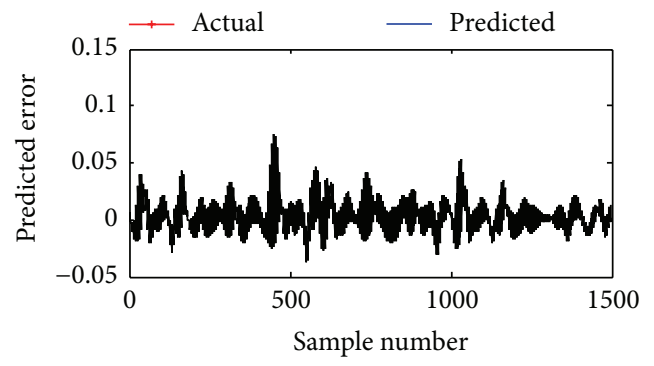

(c)
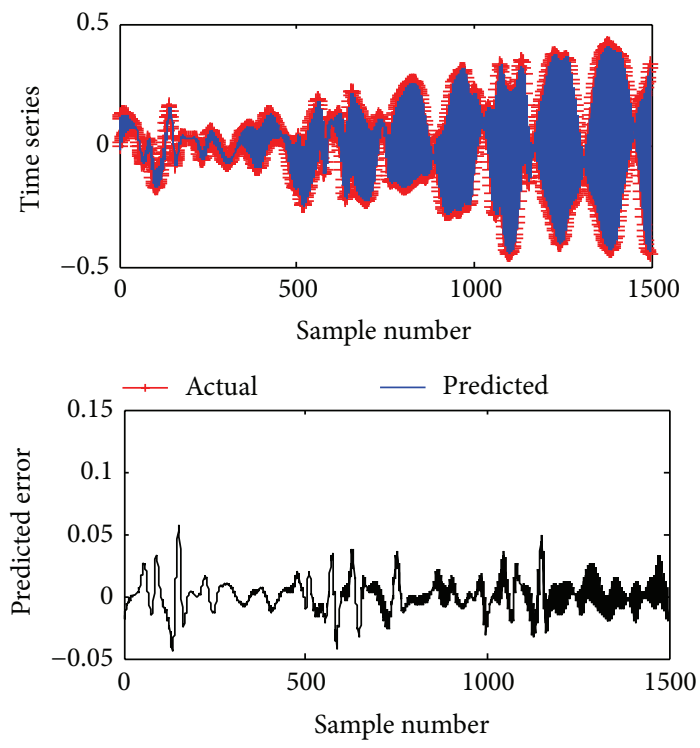

(e)

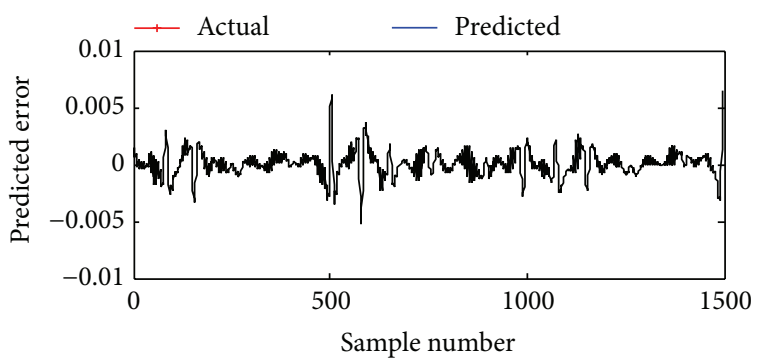

(d)
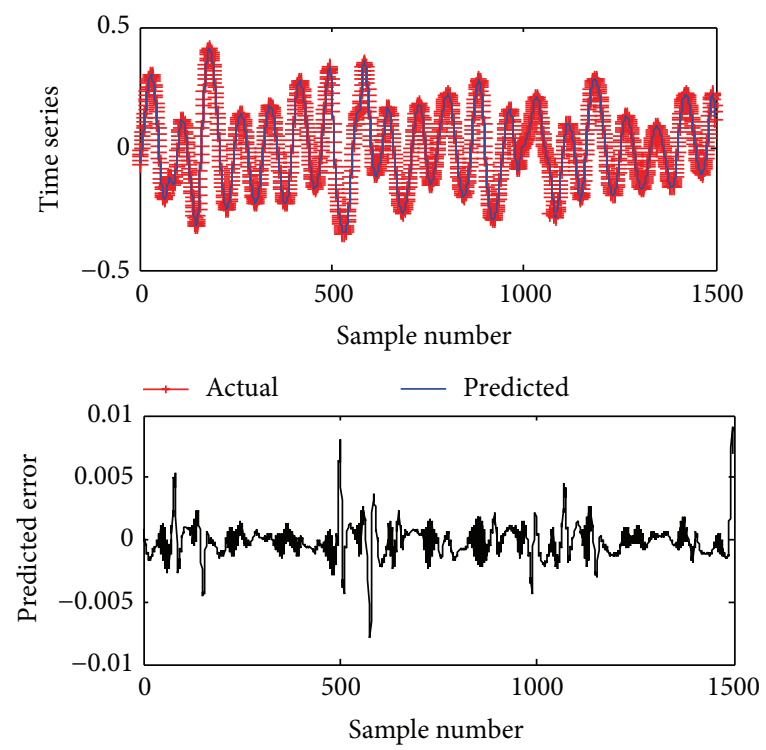

(f)

FIGURE 7: The prediction results of the chaotic vibration ((a)-(f) $Q_{11}, Q_{12}, Q_{21}, Q_{22}, Q_{31}, Q_{32}$ ). 


\section{References}

[1] P. M. Pathak, R. P. Kumar, A. Mukherjee, and A. Dasgupta, "A scheme for robust trajectory control of space robots," Simulation Modelling Practice and Theory, vol. 16, no. 9, pp. 1337-1349, 2008.

[2] M. Sabatini, P. Gasbarri, R. Monti, and G. B. Palmerini, "Vibration control of a flexible space manipulator during on orbit operations," Acta Astronautica, vol. 73, no. 4, pp. 109-121, 2012.

[3] S. A. A. Moosavian and E. Papadopoulos, "Free-flying robots in space: an overview of dynamics modeling, planning and control," Robotica, vol. 25, no. 5, pp. 537-547, 2007.

[4] T. F. P. A. T. Pazelli, M. H. Terra, and A. A. G. Siqueira, "Experimental investigation on adaptive robust controller designs applied to a free-floating space manipulator," Control Engineering Practice, vol. 19, no. 4, pp. 395-408, 2011.

[5] S. Cocuzza, I. Pretto, and S. Debei, "Novel reaction control techniques for redundant space manipulators: theory and simulated microgravity tests," Acta Astronautica, vol. 68, no. 11-12, pp. 1712-1721, 2011.

[6] W. Congqing, W. Pengfei, Z. Xin, and P. Xiwu, "Composite sliding mode control for a free-floating space rigid-flexible coupling manipulator system," International Journal of Advanced Robotic Systems, vol. 10, article 124, 2013.

[7] S. Luo and S. Ahmad, "Predicting the drift motion for kinematically redundant robots," IEEE Transactions on Systems, Man, and Cybernetics, vol. 22, no. 4, pp. 717-728, 1992.

[8] F. B. M. Duarte and J. A. T. Machado, "Motion chaos in the pseudoinverse control of redundant robots," in Proceedings of the 6th International Workshop on Advanced Motion Control, pp. 624-629, IEEE, Nagoya, Japan, 2000.

[9] A. S. Ravishankar and A. Ghosal, "Nonlinear dynamics and chaotic motions in feedback-controlled two-and three-degreeof-freedom robots," The International Journal of Robotics Research, vol. 18, no. 1, pp. 93-108, 1999.

[10] H. M. Khanlo, M. Ghayour, and S. Ziaei-Rad, "Chaotic vibration analysis of rotating, flexible, continuous shaft-disk system with a rub-impact between the disk and the stator," Communications in Nonlinear Science and Numerical Simulation, vol. 16, no. 1, pp. 566-582, 2011.

[11] R. Wang and Z. Jing, "Chaos control of chaotic pendulum system," Chaos, Solitons and Fractals, vol. 21, no. 1, pp. 201-207, 2004.

[12] S. A. Zanjani, A. H. Jafari, and A. M. Nasrabadi, "Approach to OGY control fuzzification of 2-link rigid robot arm with fixedpoints distances considering (using method similar to bellmanford algorithm)," European Journal of Scientific Research, vol. 39, no. 4, pp. 469-476, 2010.

[13] S. Dadras and H. R. Momeni, "Adaptive sliding mode control of chaotic dynamical systems with application to synchronization," Mathematics and Computers in Simulation, vol. 80, no. 12, pp. 2245-2257, 2010.

[14] C.-Q. Wang, P.-F. Wu, and X. Zhou, "Control and modeling of chaotic dynamics for a free-floating rigid-flexible coupling space manipulator based on minimal joint torque's optimization," Acta Physica Sinica, vol. 61, no. 23, Article ID 230503, 2012.

[15] S. M. Shahruz and L. R. Siva, "Suppression of chaos in a class of non-linear systems by disturbance observers," Journal of Sound and Vibration, vol. 271, no. 1-2, pp. 481-491, 2004.

[16] M. T. Arjmand, H. Sadeghian, H. Salarieh, and A. Alasty, "Chaos control in AFM systems using nonlinear delayed feedback via sliding mode control," Nonlinear Analysis. Hybrid Systems. An
International Multidisciplinary Journal, vol. 2, no. 3, pp. $993-$ 1001, 2008

[17] H. Salarieh and A. Alasty, "Control of chaos in atomic force microscopes using delayed feedback based on entropy minimization," Communications in Nonlinear Science and Numerical Simulation, vol. 14, no. 3, pp. 637-644, 2009.

[18] B. Samanta, "Prediction of chaotic time series using computational intelligence," Expert Systems with Applications, vol. 38, no. 9, pp. 11406-11411, 2011.

[19] D. Li, M. Han, and J. Wang, "Chaotic time series prediction based on a novel robust echo state network," IEEE Transactions on Neural Networks and Learning Systems, vol. 23, no. 5, pp. 787797, 2012.

[20] M. Behmanesh, M. Mohammadi, and V. S. Naeini, "Chaotic time series prediction using improved ANFIS with imperialist competitive learning algorithm," International Journal of Soft Computing and Engineering, vol. 4, no. 4, pp. 25-33, 2014.

[21] V. N. Vapnik, The Nature of Statistical Learning Theory, Springer, New York, NY, USA, 1995.

[22] S. Mukherjee, E. Osuna, and F. Girosi, "Nonlinear prediction of chaotic time series using support vector machines," in Proceedings of the 7th IEEE Workshop on Neural Networks for Signal Processing (NNSP '97), pp. 511-520, Amelia Island, Fla, USA, September 1997.

[23] W. Wang, C. Men, and W. Lu, "Online prediction model based on support vector machine," Neurocomputing, vol. 71, no. 4-6, pp. 550-558, 2008.

[24] Z. Shi and M. Han, "Support vector echo-state machine for chaotic time-series prediction," IEEE Transactions on Neural Networks, vol. 18, no. 2, pp. 359-372, 2007. 


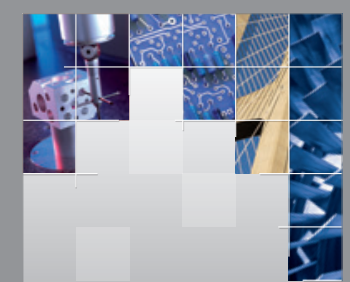

\section{Enfincering}
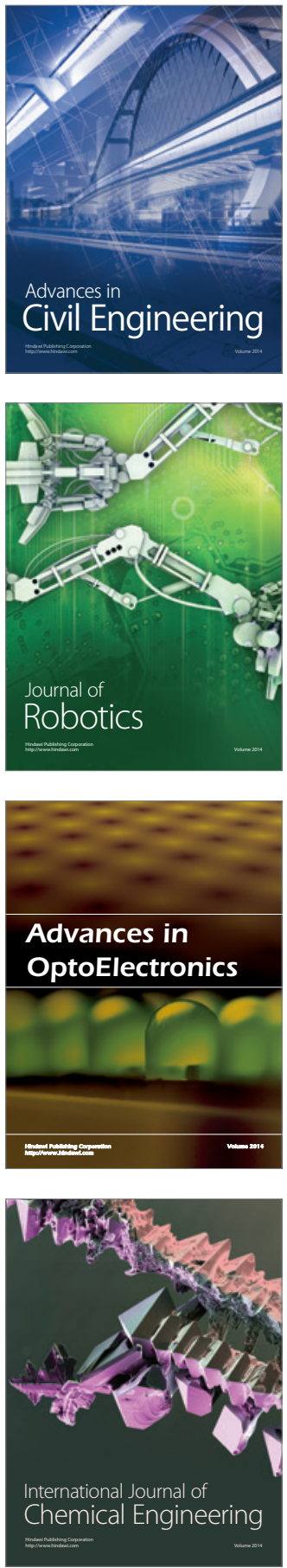

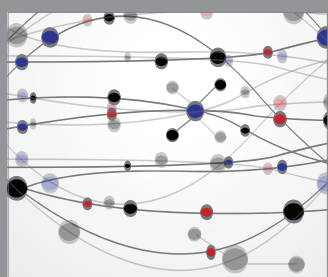

The Scientific World Journal

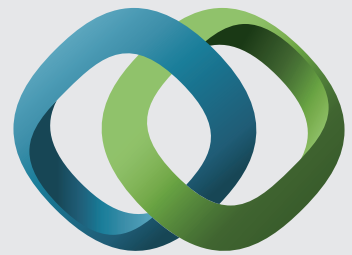

\section{Hindawi}

Submit your manuscripts at

http://www.hindawi.com
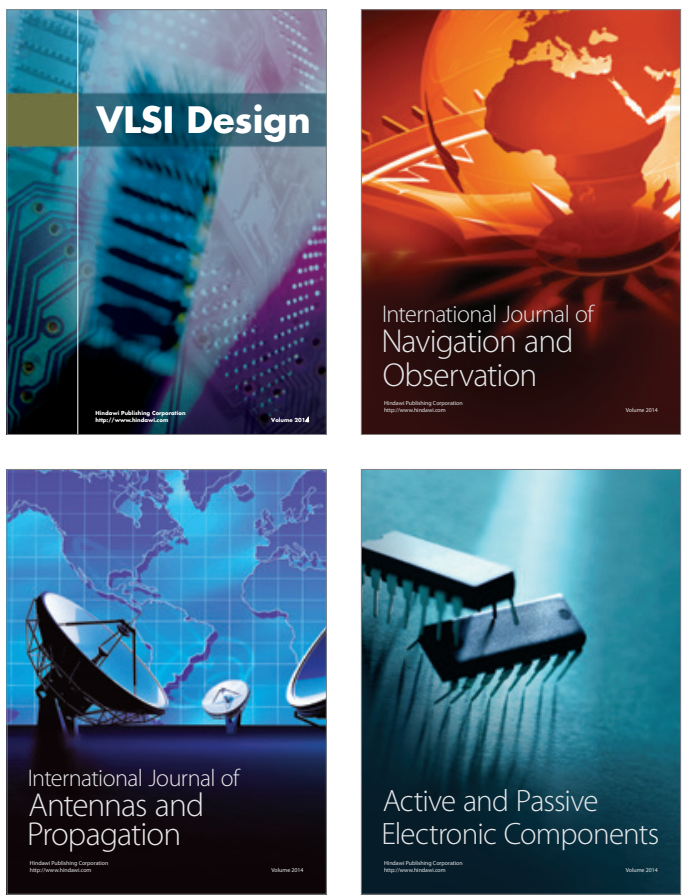
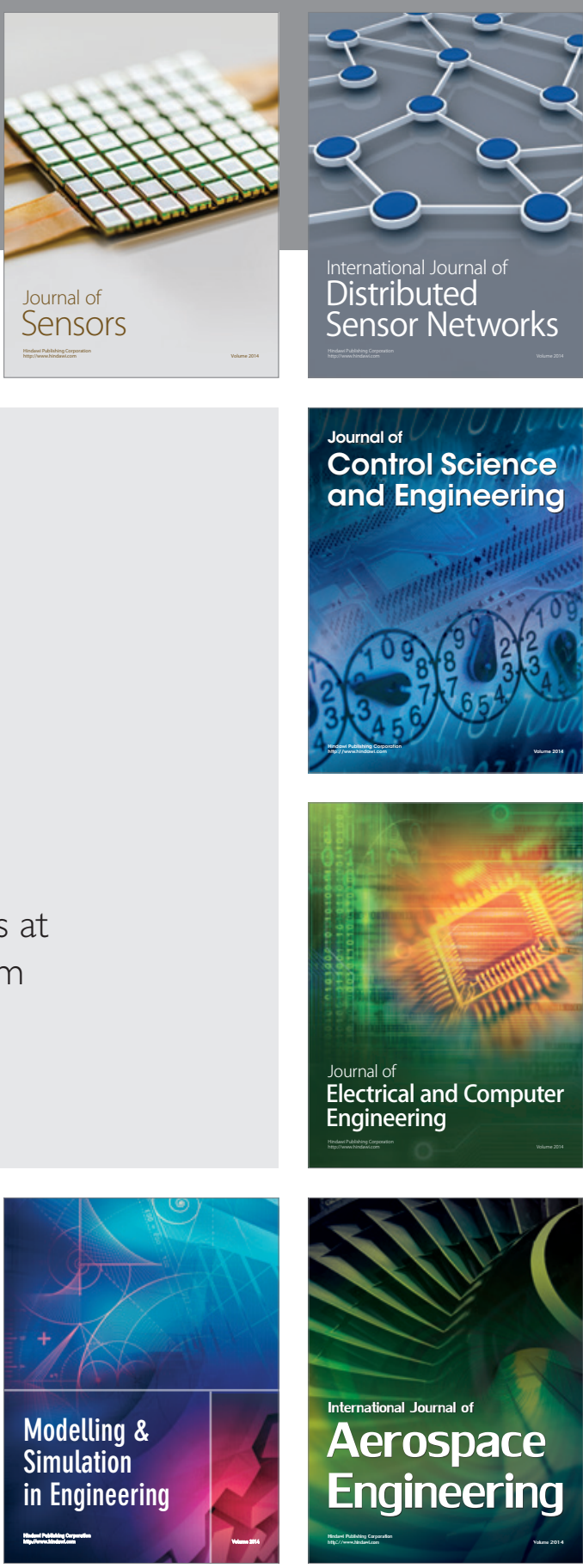

International Journal of

Distributed

Sensor Networks

Journal of

Control Science

and Engineering
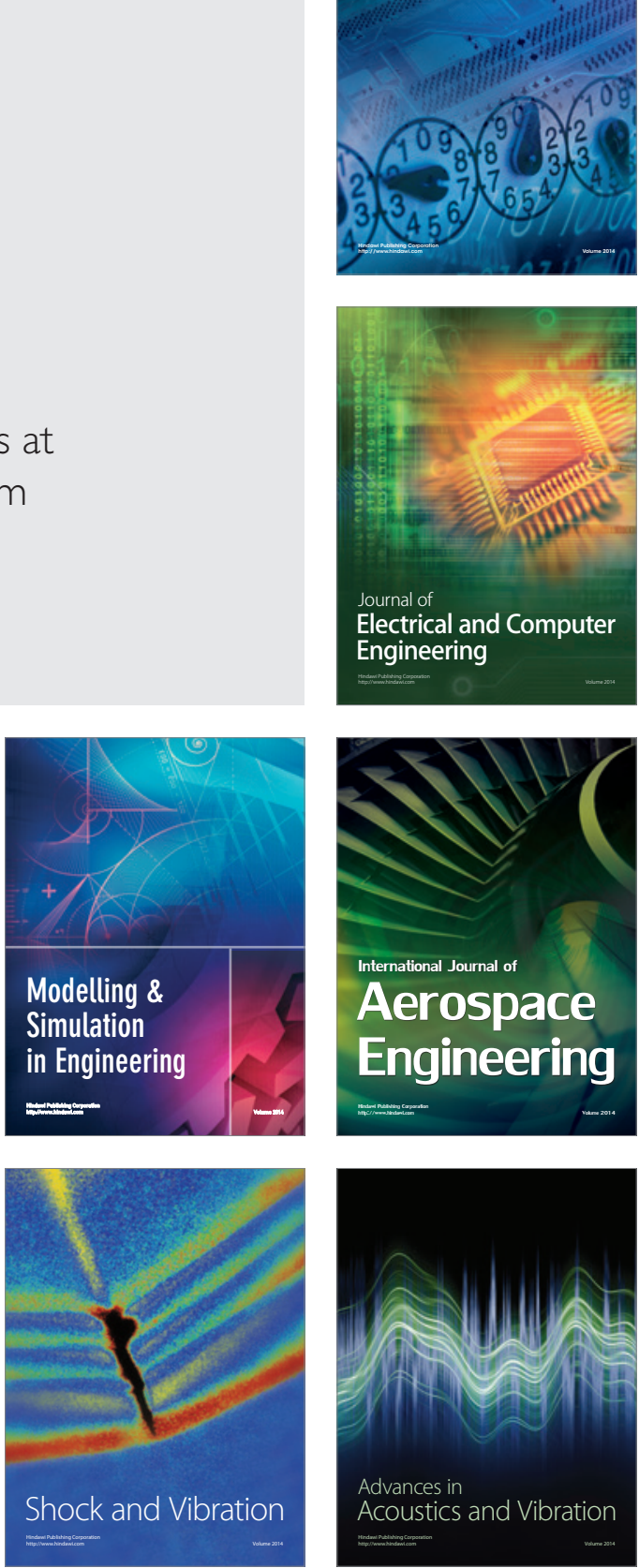